\title{
Optical loading sensor based on single polarization fiber laser incorporating an intra-cavity $\mathbf{4 5}^{\circ}$-TFG
}

\author{
Zhongyuan Sun ${ }^{* 1}$, Chengbo Mou ${ }^{1}$, Zhijun Yan ${ }^{1}$, Xiangchuan Wang ${ }^{1,2}$, Lin Zhang ${ }^{1}$ \\ ${ }^{1}$ Aston Institute of Photonic Technologies, Aston University, Birmingham, UK, B4 7ET \\ ${ }^{2}$ Institute of Optical Communication Engineering, Nanjing University, Nanjing, 210093, China
}

\begin{abstract}
We have experimentally demonstrated an active loading sensor system based on a fiber ring laser with single-polarization output using an intra-cavity $45^{\circ}$-tilted fiber grating $\left(45^{\circ}-\mathrm{TFG}\right)$. When the laser cavity fiber subjected to loading, the laser output is encoded with the load and can be measured and monitored by a power metre. A loading sensitivity as high as $0.033 /\left(\mathrm{kg}^{\circ} \mathrm{m}^{-1}\right)$ has been achieved using this laser. The experiment results clearly show that single polarization fiber laser may be developed to a low-cost high-sensitivity loading sensor system.
\end{abstract}

Keywords: Tilted fiber grating, Single polarization fiber laser, Loading sensor.

\section{INTRODUCTION}

Optical sensors for loading measurement based on fiber Bragg grating (FBG) ${ }^{[1,2]}$, long period grating (LPG) ${ }^{[3]}$, and tilted fiber grating ${ }^{[4]}$ have been extensively studied due to their small size, lightness, long-term stability, immunity to electric-magnetic interference and most of all, the multiplexing capability for sensor network. To function as loading sensors, the majority of these gratings exhibited a pronounced polarization mode split effect resulting from the birefringence induced by the transverse loading. Lin Zhang et al ${ }^{[3]}$ reported an optical fiber load sensor based on an LPG fabricated in conventional and Hi-birefringence fiber. Rui et al ${ }^{[5]}$ experimentally demonstrated a directional transverse loading sensor based on an $81^{\circ}$-TFG.

Most fiber loading sensors proposed so far are based on passive operation with expensive spectrometer for signal demodulation. External light source will also be required in such a type of sensors. In this paper, we report a loading sensor using single polarization fiber laser incorporating an intra-cavity $45^{\circ}-\mathrm{TFG}$. The proposed laser sensor system is based on monitoring the output laser power which is at low cost compared to the passive sensor demodulation.

\section{STRUCTURE AND POLARIZATION PROPERTY OF $\mathbf{4 5}^{\circ}$-TFG}

$45^{\circ}$-TFG is a novel type of gratings with a series of index modulation planes that are tilted at 45 degree with respect to the fiber axis. Theoretically, when light propagates through the $45^{\circ}-\mathrm{TFG}$, the s-polarized light radiates out of the fiber core and the p-polarized light propagates through the fiber without any loss. It is pointed out that the strongest coupling wavelength for a $45^{\circ}$-TFG is at ${ }^{[6]}$ :

$$
\lambda_{\text {strongest }}=2 n \Lambda_{G} \operatorname{Cos} 45^{\circ}
$$

Where $n$ is the effective refractive index of core mode and $\Lambda_{G}$ is the grating period along the fiber.

Detailed description of the fabrication of the $45^{\circ}-\mathrm{TFG}$ is described elsewhere [6]. The effective length of $45^{\circ}$-TFG used in this laser sensing experiment is around $\sim 25 \mathrm{~mm}$. Figure 1(a) shows the polarization dependent loss (PDL) spectral response of the $45^{\circ}$-TFG which was immersed into an index matching gel and measured by a commercial optical analysis system (LUNA optical vector analyser). It can be seen from the figure that PDL is about $15 \mathrm{~dB}$ around wavelength $1550 \mathrm{~nm}$ and dropped to $12 \mathrm{~dB}$ at $1600 \mathrm{~nm}$. Figure $1(\mathrm{~b})$ shows the image of a $45^{\circ}-\mathrm{TFG}$ structure in the fiber core that was taked by a $100 \mathrm{x}$ microscope.

*Corresponding author: sunz5@aston.ac.uk

23rd International Conference on Optical Fibre Sensors, edited by José Miguel López-Higuera,

Julian Jones, Manuel López-Amo, José Luis Santos, Proc. of SPIE Vol. 9157, 91577H

(C) 2014 SPIE · CCC code: 0277-786X/14/\$18 - doi: 10.1117/12.2058482 

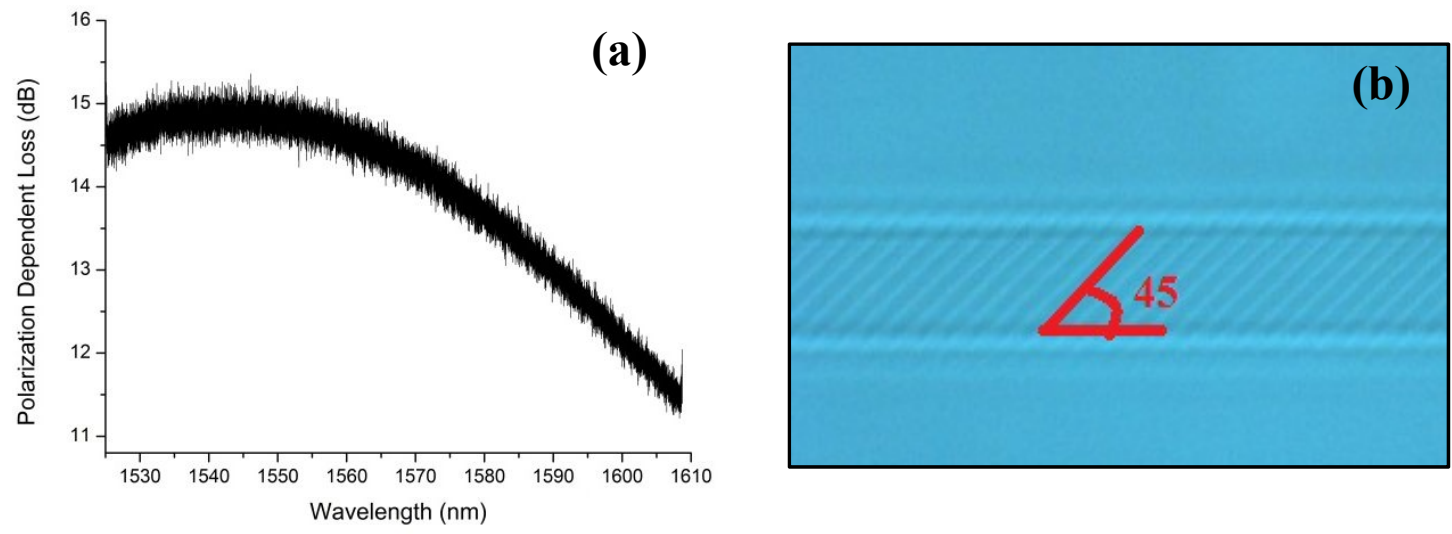

Figure 1 (a) The PDL spectral response of the $45^{\circ}$-TFG. (b) Microscopic image of a $45^{\circ}$ - TFG taken under a $100 x$ oil immersion objective lens.

\section{EXPERIMENTAL SETUP, OPERATION PRINCIPLE AND RESULTS}

\subsection{Experimental setup of single polarization laser system based on $45^{\circ}$-TFG}

The experimental setup of single polarization ring laser based on $45^{\circ}$-TFG is illustrated in Figure 2(a). A 4-metres length of Erbium-doped fiber (EDF) was inserted in the ring cavity and pumped by a grating stabilized $976 \mathrm{~nm}$ laser diode (LD) which can provide up to $200 \mathrm{~mW}$ pump power though a 980/1550 wavelength division multiplexing (WDM) coupler. A 10:90 coupler is employed to couple $10 \%$ of laser light out of the laser cavity. A uniform FBG with reflectivity larger than $90 \%$ and $3-\mathrm{dB}$ bandwidth of $0.19 \mathrm{~nm}$ at the wavelength of $1550.0 \mathrm{~nm}$ is incorporated in the cavity through an optical circulator, which is used to define the laser seeding wavelength while maintaining indirection oscillation. A $45^{\circ}-\mathrm{TFG}$ is inserted between the circulator and the polarization controller (PC) to achieve single polarization function, as it can optimize the cavity birefringence. Figure 2(b) provides a typical output of the ring laser measured by an optical spectrum analyser (OSA).
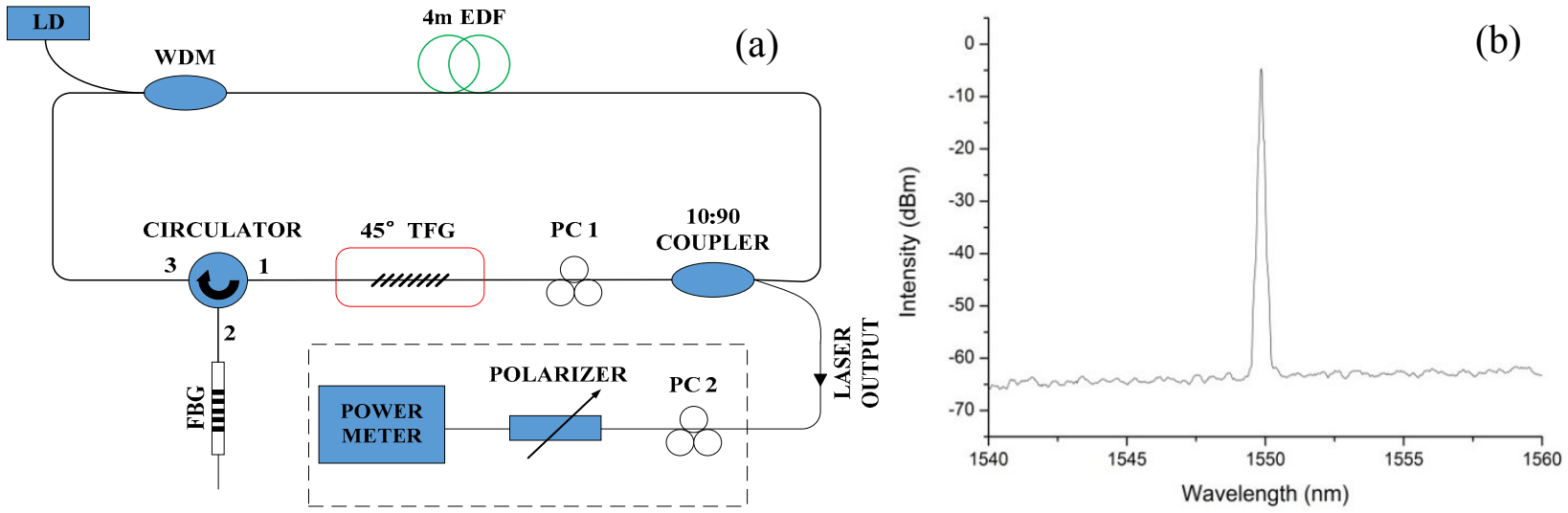

Figure 2 (a) Schematic diagram of the single polarization fiber ring laser structure. The polarization extinction ratio of the laser output is measured using the setup shown in dashed line box. (b) Typical output spectrum of the fiber laser.

Without $45^{\circ}$-TFG in the ring cavity, the fiber ring laser output has a degree of polarization (DOP) of only about $\sim 19.7 \%$, which suggests that the laser output is almost un-polarized. After inserting the $45^{\circ}$-TFG into the laser cavity, the DOP of laser output significantly increases to $\sim 99 \%$, which clearly indicates that the output of the laser is highly polarized and almost single polarization. The slope efficiency of the fiber laser has also been examined before and after incorporating the $45^{\circ}$-TFG into the cavity. Figure 3(a) clearly shows the slope efficiency decreased from $11.93 \%$ to $8.06 \%$ after the $45^{\circ}$-TFG had been inserted into the laser cavity. This is owing to the $45^{\circ}$-TFG induced PDL-related total loss in the laser 
cavity. We also measured the stability of the fiber ring laser output with the $45^{\circ}$-TFG in the cavity. Figure 3 (b) displays that typical output spectra of fiber ring cavity laser measured over half an hour at a 5 minutes interval. No noticeable spectral variation is shown in the figure.
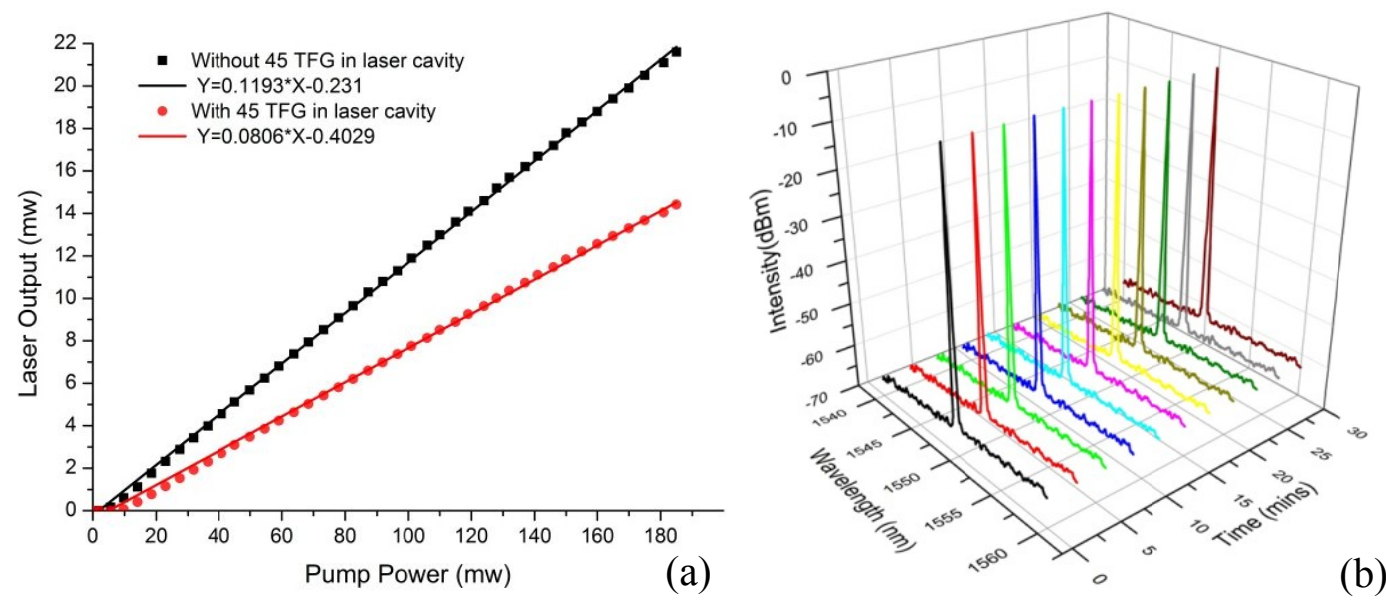

Figure3 (a) Slope efficiency of the fiber ring laser with and without the $45^{\circ}$-TFG. (b) The stability of fiber ring cavity laser output spectra measured over half an hour at the laboratory condition.

\subsection{Loading sensing principle}

As a result of the polarizing property of the $45^{\circ}-\mathrm{TFG}$, the proposed fiber laser cavity could eliminate one polarization state thus giving out single polarization output. Therefore, only the light with appropriate polarization state that matches the transmission axis of the $45^{\circ}$-TFG will have the maximum lasing efficiency in the cavity. Any perturbation that changes the intra-cavity polarization state will lead to extra loss, hence a reduction in the output power. Due to photo-elastically induced birefringence introduced when transverse force applied onto the single mode silica fibre, the applied loading can thus alternate the intra-cavity polarization state of a fibre laser. The $45^{\circ}$-TFG functions as a polarization filter in laser cavity, therefore any variation of birefringence or polarization in the cavity will lead to the laser output power change. This is the principle of our fiber laser based loading sensor.

\subsection{Experimental setup of laser loading sensor and results}

The schematic of laser loading experiment is shown in Figure 4(a). The laser structure is the same as the one illustrated in Figure 2(a); the dotted circle in Figure 4(a) indicates the sensing fiber section. The plastic coating of the sensing fiber has been removed to reduce the buffer effect. The loading setup is shown in the dotted box, which was implemented by laying the sensing fiber and a dummy fiber (SMF-28) between two flat-surface aluminium plates. In the experiment, we gradually increased the load on the top of the plate with active loading length $31 \mathrm{~mm}$. The laser output was continuously monitored by a power meter.

Before applying the transverse load, we maximized the laser output power by adjusting the PC to guarantee the laser working in an optimized condition. We then applied the transverse load to the sensing fiber in cavity from 0 to $6.4 \mathrm{~kg}$ in an elevation step of $0.1 \mathrm{~kg}$. The laser output power changed with loading weight variation due to the alteration of intra-cavity polarization state. The measured output optical power changes against the loading weight on two different active loading lengths are plotted in Figure 4(b). It can be seen that the normalized output power is varied with increasing load in a similar trigonometric function for a full circle change of the polarization state. However, in practical application, a linear relationship is maybe more desirable. Therefore, taking the quasi-linear range from $32 \mathrm{~kg} / \mathrm{m}$ to $61 \mathrm{~kg} / \mathrm{m}$ as an example, we can estimate that the loading sensitivity is approximately $0.033 /\left(\mathrm{kg} \cdot \mathrm{m}^{-1}\right)$. Compared with TFG based loading sensor reported before [5], the sensitivity of our active loading sensor has been increased significantly. 

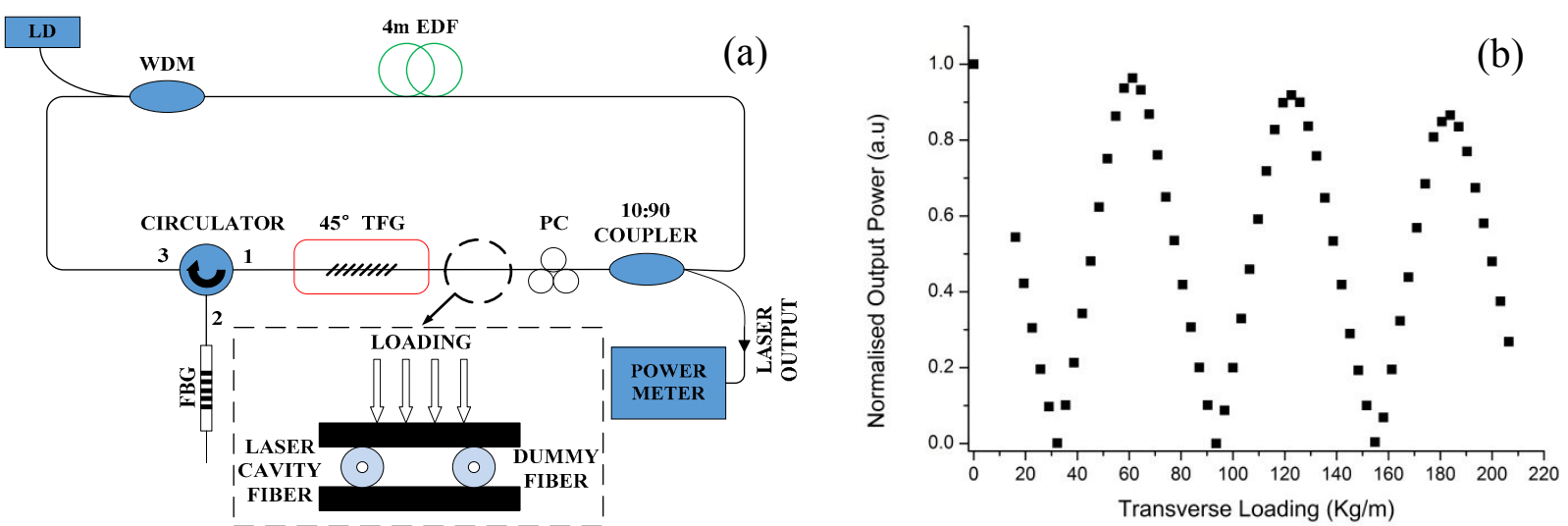

Figure 4 (a) Schematic of the fiber laser loading sensor incorporating an intra-cavity $45^{\circ}$-TFG. (b) Measured output power change against loading weights.

\section{CONCLUSION}

In summary, we have demonstrated a single polarisation fiber laser incorporating an intra-cavity $45^{\circ}$-TFG for loading sensing. The loading sensing based on this active configuration has shown a significantly increased sensitivity compared with the TFG based passive one. The fiber ring laser sensor system also benefits from low cost signal demodulation method.

\section{REFERENCES}

[1] Abe I., Kalinowski H. J., Frazão O., Santos J. L., Nogueira R. N. and Pinto J. L. ,"Superimposed Bragg gratings in high-birefringence fiber optics: three-parameter simultaneous measurements," Measurement Science \& Technology 15(8), 1453-1457 (2004).

[2] Shu X., Karen C., Ian F., Sugden, K., Gillooly, A., Zhang L. and Bennion I., "Highly sensitive transverse load sensing with reversible sampled fiber Bragg gratings," Applied Physics Letters 83(15), 3003-3005 (2003).

[3] Zhang L., Liu, Y. , Everall, L., Williams, J.A.R. and Bennion, I., "Design and realization of long-period grating devices in conventional and high birefringence fibers and their novel applications as fiber-optic load sensors," Ieee Journal of Selected Topics in Quantum Electronics 5(5), 1373-1378 (1999).

[4] Jiang Q., D.B. Hu, "Micro displacement Sensor Based on Tilted Fiber Bragg Grating Transversal Load Effect," Ieee Sensors Journal 11(9), 1776-1779 (2011).

[5] Suo R., Chen X., Zhou K., Zhang L. and Bennion I., "In-fiber directional transverse loading sensor based on excessively tilted fiber Bragg gratings," Measurement Science \& Technology 20(3), 1-6 (2009).

[6] Yan Z., Mou C., Zhou K., Chen X. and Zhang L., "UV-Inscription, Polarization-Dependent Loss Characteristics and Applications of 45 Tilted Fiber Gratings," Journal of Lightwave Technology 29(18), 2715-2724 (2011). 